\title{
The First Four Million Years of Human Evolution
}

Alan Walker and Chris Stringer (eds.)

Special Issue of the Philosophical Transactions of the Royal Society B (Biological Sciences) Vol. 365: 3263-3410, 2010, £47.50.

ISSN: 0962-8436.

\section{Reviewed by SUSAN CACHEL}

Department of Anthropology, Rutgers University, 131 George Street, New Brunswick, NJ 08901-1414, USA; Cachel@rci.rutgers.edu

$\mathrm{T}$ his volume is the result of a two-day meeting that took place at the Royal Society of London in 2009. Alan Walker and Chris Stringer organized the conference and also edited the volume. The meeting was designed as a commemoration, because it coincided with the 200th anniversary of Charles Darwin's birth, and the 150th anniversary of the publication of The Origin of Species. The Royal Society was also about to celebrate its 350th anniversary in 2010, and was initiating a series of programs to highlight advances in science. This meeting was meant to showcase the interdisciplinary nature of paleoanthropology - which did not exist in Darwin's time - and to display recent methodological advancements in the study of human evolution.

The editors state in the Introduction that they are focusing on the time span from 6-2 mya, in order to elucidate the morphology, ecology, and behavior that lie beneath and trigger the origin of genus Homo. The papers are presented in chronological order and in anatomical sections, working from the postcranium, to the dentition, and to the cranium. Papers on sexual dimorphism, diet, and ontogenetic rates are based on the dentition. Notably absent is any paper on the brain or brain evolution, though, given the early time range, this may be understandable. However, it is a sign of the times that no comment at all is made of encephalization or relative brain size. Bipedalism now triumphs as the hallmark of human evolution.

McGrew begins the volume with a review of work published since 2005 on wild common chimpanzees from eight study sites with completely habituated animals. He attempts to recreate the behavior of the Last Common Ancestor of chimpanzees and hominids. He is certain that the behavior of the Last Common Ancestor can be retrieved from chimpanzees, and can then be used directly to address crucial topics in human evolution. These topics are technology, diet, shelter, and ranging and foraging. McGrew believes that discussions about the most appropriate way to model human evolution using living primates "muddied the waters" (p. 3267). He adamantly rejects, although he does not address, the seminal critique of Sayers and Lovejoy (2008). These authors argued that chimpanzees are not especially valuable as models for human evolution, and that other non-human primate species (such as members of genus Cebus) can be more suitable. Because the eight chimpanzee study sites cross a range of habitats, McGrew seeks behaviors that are known to be invariant across habitats. He opposes these to other behaviors that demonstrate vast or slight differences between habitats. He believes that this allows one to identify primitive traits of the Last Common Ancestor and derived traits of hominids. McGrew argues that percussive hammer and anvil use during chimpanzee nut-cracking foreshadows the protoOldowan. However, chimpanzees do not produce flakes or debitage, but microshatter. Their "artifacts" can be distinguished from true, humanly manufactured artifacts. McGrew admits that there is no evidence of active teaching in chimpanzees. Finally, chimpanzees do not range as far as hominids do, because their locomotion is more costly than bipedalism, and there is no sign of long-distance transport of resources.

Stone et al. examine complete mtDNA sequences in eight specimens of Pan troglodytes in order to infer divergence times between chimpanzee species and subspecies. Their results demonstrate that common chimpanzees have about four times the nucleotide diversity that living humans do. Female biased dispersal and bottlenecks affect the mtDNA results. Chimpanzees and bonobos separate late in time, between 2.1-1.5 mya, which renders their behavioral and ecological differences fairly remarkable.

Lovejoy and McCollum reconstruct the most ancient form of hominid locomotion, focusing on evolution of the vertebral column and the pelvis, as well as on newly described fossils of Ardipithecus ramidus. The paper argues strongly against the consensus opinion that locomotion in African apes reveals anything about the origins of bipedalism. Hominids did not evolve from knuckle-walkers. The bent-hip, bent-knee gait observed in bipedal chimpanzees or gorillas is eschewed as being significant. This gait is caused by a short, osteologically rigid lumbar region, and iliac blades that are extended cranially to hinder movement of the posterior lumbar vertebrae. African apes must therefore flex their hips and knees to orient their center of mass over the point of contact with the ground. African apes have only three to four lumbar vertebrae, but Australopithecus and early Homo erectus specimens have six lumbar vertebrae. The short, stiff lumbar region of the African apes reflects their specialization for suspensory locomotion and vertical climbing. Ardipithecus, on the other hand, retains a more generalized axial skeleton, and its intrinsically stiff foot, adapted for walking above branches, is also suitable for walking on the ground.

Crompton et al. deal with how aspects of arboreal and terrestrial life affect the emergence of bipedalism. They agree with Lovejoy and McCollum that any form of locomotion associated with bent knees and bent hips, such as 
knuckle-walking or vertical climbing, is unlikely to have given rise to bipedalism. Instead, they consider that bipedalism arises in the trees, as arboreal animals use their hands to maintain an upright posture. They refer to this as "compressive orthogrady." Crompton et al. thus argue that the Last Common Ancestor of humans and the African apes had a form of locomotion not seen in any living species. They disagree with Lovejoy and McCollum about locomotion in Ardipithecus. They reconstruct Ardipithecus as engaging in "compressive orthogrady" in the trees, rather than being an arboreal quadruped, like an Old World monkey. Crompton et al. also note that the large body size of Ardipithecus makes this reconstruction more likely.

Brunet found and described two new taxa from northern Chad-Australopithecus bahrelghazali and Sahelanthropus tchadensis. He now discusses the implications of finding these discoveries not only west of the African Rift Valley, but also finding them dispersed very far from the Rift Valley. Brunet also envisions the late Miocene paleoenvironments of these taxa, noting evidence for mosaic habitats that included woodland. Haile-Selassie discusses 3.8-3.6 mya old hominid fossils from the Woranso-Mille area of the Afar, Ethiopia. These fossils exhibit a mosaic of features that are characteristic of both Australopithecus anamensis and Australopithecus afarensis. Haile-Selassie therefore argues that anagenesis is occurring- "A. anamensis" simply represents earlier and more primitive versions of the same lineage. A. afarensis has taxonomic priority. Yet, because the scientific literature can be irretrievably confused, the fossils from Woranso-Mille may need to be referred to as $A$. anamensis. This problem epitomizes anagenesis - the first and last members of an evolving lineage can be well differentiated; however, fossils in the middle of the sequence show a mixture of traits that are difficult to separate. Ward et al. discuss the evolution of the dentition in the $A$. anamensis- $A$. afarensis lineage, and thus confirm the existence of anagenesis. Changes occur in the anterior dentition, which exhibits less extreme wear in $A$. afarensis. The tooth rows become more parabolic, the mandibular symphysis becomes more robust, and the molar crowns increase in height. Canine roots are shortened, and the canines become less dimorphic in $A$. afarensis. The authors suggest that $A$. afarensis has greater masticatory loads on the postcanine dentition, but greatly reduced use of the anterior dentition.

Ungar et al. use dental microwear to study diet in Australopithecus anamensis and Australopithecus afarensis. Microwear textures are not complex; they resemble Australopithecus boisei in complexity. The authors infer that food items were not hard, brittle, or tough. These hominid species were not hard-object feeders. Microwear textures in Australopithecus africanus and Australopithecus robustus are very different. Lee-Thorp et al. use stable isotopes from tooth enamel to study diet in South African australopithecine species and East African Australopithecus boisei. All of the species examined show a reliance on $\mathrm{C}_{4}$ food resources. Variability within single teeth indicates seasonal dietary shifts. The dependence on $\mathrm{C}_{4}$ food and marked seasonal variation in diet is radically different from the chimpan- zee condition. The high relative proportion of $\mathrm{C}_{4}$ foods in hominid diets persists for over a million years, despite significant habitat changes. Ardipithecus ramidus does not have this dietary configuration, and appears to have avoided $\mathrm{C}_{4}$ foods, although they were present in its environment. The authors argue that a reliance on $\mathrm{C}_{4}$ foods finally emerges between 4-3 mya. This dietary pattern then becomes a fundamental criterion of later hominids.

Reno et al. study the postcranial remains of Australopithecus afarensis, and contend-contrary to general opinion-that sexual dimorphism was greatly reduced in this species, and resembled the degree of dimorphism observed in modern humans. Their original sample size, which generated this daring conclusion, is expanded by a dozen more specimens. Reno et al. attribute the decline in sexual dimorphism to the advent of monogamy-males are provisioning pair-bonded females with vertebrate meat and fat. This results in increased reproductive success, which triggers a rise in hominid population numbers, and leads to a fundamental differentiation between hominids and their closest primate relatives. However, as observed above, Lee-Thorp et al. noted the avoidance of $\mathrm{C}_{4}$ foods (meat from ungulates consuming grasses) in Ardipithecus ramidus.

Kimbel and Rak compare cranial base morphology in fossil hominids, focusing particularly on a newly reconstructed female skull of Australopithecus afarensis from Hadar, Ethiopia. This skull (A.L. 822-1) is beautifully illustrated at about 45 percent natural size in Figures 1, 2, and 4. Kimbel and Rak discover that relative brain size does not affect the position of the foramen magnum, because bipedality is the most important variable affecting its location. The foramen magnum is positioned anteriorly in both australopithecines and genus Homo, although it does not incline forward in the australopithecines. Strangely, the morphology of the cranial base in adult Australopithecus afarensisand possibly other australopithecines-shows sexual dimorphism. Female specimens have a narrow cranial base, a steep nuchal plane, and high nuchal lines that simulate an ape-like condition. Male specimens are more derived, with a wide cranial base, a horizontal nuchal plane, and low nuchal lines. Kimbel and Rak argue that this dimorphism has an ontogenetic basis-young males exhibit a female-like morphology before developing the more derived condition observed in mature males. Spoor et al. support the existence of the controversial species Kenyanthropus platyops, based on the 3.5 my old type specimen KNM-WT 40000. A diagnostic trait of this species is its flat and orthognathic maxilla, which supposedly links it uniquely to the origin of genus Homo. Yet, White (2003) argued that matrix expanding in cracks between fossil fragments had seriously distorted the maxilla-a conclusion that has become the consensus opinion. Spoor et al. attempt to rebut White through landmark analysis. Yet, unfortunately for the authors, a plethora of matrix-filled cracks in the type specimen is highlighted in Figure 2, which renders their attempt to rebut White untenable.

Dean exhaustively reviews maturation rates in early hominids based on tooth microstructure. He discovers evi- 
dence that dental development in early hominids might resemble that of gorillas, rather than chimpanzees. Dean also describes extensive dental wear in some infant and juvenile australopithecines, which implies some intake of adult foods even before the young were weaned. Alternatively, youngsters may have been weaned at a very early age.

In summary, this volume is a panoply of recent studies on the earliest hominids. It highlights new investigative techniques, and demonstrates how fully these techniques can illuminate aspects of early hominid life, such as locomotion, diet, and maturation. Two features that link the various papers together are that brain evolution is not mentioned, and, except for McGrew's paper, the primacy of chimpanzees in models of human evolution is downplayed. This approach is very different from the one that characterized Darwin's time. The volume thus epitomizes the radically different changes that have occurred in the study of human evolution over the last 150 years. Does the volume fulfill the hopes of its editors? Does it illuminate the morphology and behavior that underlie the origin of genus Homo? Yes, but it is clear that the transition to Homo was gradual. Leaving aside the evidence of archaeology, no shattering events or breakthroughs herald the earliest members of genus Homo. Rather, the knick-point occurs with the later australopithecines, whose locomotion, diet, and ranging behavior differ from that of the earliest hominids.

\section{REFERENCES}

Sayers, K. and Lovejoy, C.O. 2008. The chimpanzee has no clothes. A critical examination of Pan troglodytes in models of human evolution. Current Anthropology 49, 87-114.

White, T.D. 2003. Early hominids-diversity or distortion? Science 299, 1994-1997. 